\title{
Microwave Promoted Sodium Acetate Catalyzed One Pot Synthesis of Highly Functionalized Pyridine Derivatives
}

\author{
M. S. PANDHARPATTE ${ }^{1}$, HASSAN.A.OSMAN ${ }^{2}$, \\ ABU-BKER.M.OSMAN ${ }^{3}$ and G. M. NAZERUDDIN* \\ *Department of Chemistry, Poona College of Arts, Science \& Commerce, Pune, India \\ ${ }^{1}$ Department of Chemistry, Nowrosjee Wadia College of Arts and Science, Pune, India \\ ${ }^{2}$ Department of Chemistry Nyala University, Nyala, Sudan \\ ${ }^{3}$ Department of Chemistry, Science and Arts College for girls (Suratabidha), Saudi Arabia \\ hassanahmed94@yahoo.com
}

Received 26 May 2016 / Accepted 26 June 2016

\begin{abstract}
A simple and efficient microwave promoted one pot synthesis of highly functionalized pyridine derivatives using sodium acetate as a catalyst via three component condensations of aldehydes, malonitrile and thiols is described. It is an efficient and promising synthetic protocol to build the highly functionalized pyridine derivatives.
\end{abstract}

Keywords: Multi-component reaction, Highly functionalized pyridines derivatives, Sodium acetate

\section{Introduction}

Multicomponent reactions (MCRs) constitute an especially attractive synthetic strategy since they provide easy and rapid access to large libraries of organic compounds with diverse substitution patterns ${ }^{1,2}$. As MCRs are one-pot reactions, they are easier to carry out than multi-step syntheses. The developing of new MCRs and improving known multi-component reactions are an area of considerable current interest. One such reaction is the synthesis of pyridine. Coupled with high-throughput library screening, this strategy was an important development in the drug discovery in the context of rapid identification and optimization of biologically active lead compounds. Among them, 2-amino-3,5-dicarbonitrile-6-sulfanylpyridines exhibit various pharmacological activities and are useful as anti-hepatitis B virus ${ }^{3}$, antiprion $^{4}$, antibacterial ${ }^{5}$, anti-cancer agents ${ }^{6}$ and as potassium channel openers for treatment of urinary incontinence ${ }^{7}$. Moreover, some of these compounds were found to be highly selective ligands for adenosine receptors ${ }^{8}$, implicated Parkinson's disease, hypoxia/ischemia, asthma, kidney disease and epilepsy?. 
Chem Sci Trans., 2017, 6(1), 1-7

A three-component condensation of aldehyde, malononitrile and thiol is one of the most prominent existing procedures used for the synthesis of 2-amino-3, 5-dicarbonitrile6-thio-pyridines. Generally, this condensation has been carried out under basic conditions using various bases such as, $\mathrm{Et}_{3} \mathrm{~N}, \mathrm{DABCO}$, piperidine ${ }^{10}$, morpholine, thiomorpholine, pyrrolidine, $N, N$-DIPEA, pyridine, 2,4,6-collidine, DMAP, aniline, $N$ methylaniline, $N, N$-dimethylaniline and $N, N$-diethylaniline ${ }^{10}$ and $\mathrm{DBU}^{11}$. Moreover, basic ionic liquid 1-methyl-3-butylimidazolium Lewis hydroxide, that is, [bmim] $\mathrm{OH}^{12}$ and using a variety of Lewis acids such $\mathrm{ZnCl}_{2}, \mathrm{AlCl}_{3}, \mathrm{FeCl}_{3}, \mathrm{I}_{2}, \mathrm{Cu}(\mathrm{OTf})_{3}, \mathrm{InCl}_{3}$ and $\mathrm{BF}_{3} \mathrm{Et}_{2} \mathrm{O}^{13}$. However, most of these methods suffer by the formation of inevitable side products, which results in lower yield of desired product with long reaction time. Microwave promoted organic reactions has rapidly gained popularity as it accelerates a variety of synthetic transformations and is particularly eco-friendly with of short reaction time and high yield. So, development of an improved synthetic protocol for the generation of pyridine-3,5-dicarbonitriles for lead optimization is of considerable interest. Keeping the medicinal values of pyridine-3, 5-dicarbonitriles in mind, we considered it necessary to develop an efficient high yielding synthetic protocol for the synthesis of this class of compounds.

\section{Experimental}

All reagents were purchased from Merck and Loba and used without further purification.

\section{Apparatus}

Melting points were measured in open capillary and are uncorrected. The products were characterized by IR spectra, ${ }^{1} \mathrm{H}$ NMR and ${ }^{13} \mathrm{C}$ NMR. IR spectra were recorded on PerkinElmer FT-IR-1710 instrument. ${ }^{1} \mathrm{H}$ NMR was recorded on Bruker MSL-300 MHz and BrukerMSL-200 MHz instrument using TMS as an internal standard. Elemental analyses were determined by an elemental analyser (CHNS-O, EA 1108-elemental analyser, Carlo Erba instruments). Microwave oven was used LG MOD-MG-1742WE with 230V, $2450 \mathrm{MHz}$ and $700 \mathrm{~W}$ maximum.

\section{General microwave procedure for synthesis of polysubstituted pyridines}

Aldehyde (1 mmol), malononitrile $(2.1 \mathrm{mmol})$, thiophenol $(1 \mathrm{mmol})$, anhydrous methanol $(1.5 \mathrm{~mL})$ and sodium acetate $(12 \mathrm{~mol} \%)$ were mixed and placed under microwave irradiation at $280 \mathrm{~W}$ power. After the completion of reaction monitored by TLC, the reaction mixture was cooled and precipitate formed was filtered and recrystallized from acetonitrile/methanol to yield the pure product.

\section{Results and Discussion}

In view of the potential medicinal importance of the products and considering the limitations of the existing methods, we have investigated a sodium acetate $(10 \mathrm{~mol} \%)$ catalyzed, one-pot, simple and efficient procedure for the rapid construction of substituted pyridines via a three component reaction of aldehydes $(1 \mathrm{mmol})$, malononitrile $(2.1 \mathrm{mmol})$ and thiophenol $(1 \mathrm{mmol})$ or 2-mercapto ethanol $(1 \mathrm{mmol})$ in methanol under microwave irradiation $(62-92 \%)$ (Scheme $1 \& 2$ ).

In the absence of catalyst the reaction was slow and product formed in traces. In the next step, we have screened different acidic, basic and phase transfer catalyst mention (Table 1). In comparison with these, sodium acetate proved to be almost efficient catalyst that gave higher yield within 3 min (Table 2). Sodium acetate plays a complex role in 
accelerating the coupling reaction and thus promotes the formation of products. To investigate the reaction in detail, it was carried out in various solvents, the results are depicted in Table 3, also investigated the affect of concentrations of catalyst (Table 4). To evaluate the efficiency of this methodology, various substituted aromatic and heteroaromatic aldehydes with either electron-donating or electron-withdrawing groups were used and it is found that the reaction underwent smoothly and gave the products in excellent yields. Possible mechanism of the reaction is depicted in Scheme 3.<smiles>[R]CO[N+]([O-])(OC)O[Na]</smiles>

Scheme 1. Synthesis of highly functionalized pyridine derivatives using thiophenol<smiles>[R][R]1ccccc1-c1c(N)nc(SCCO)c(C#N)c1C(N)C(C)=O</smiles>
$\mathrm{R}=\mathrm{Cl}, \mathrm{OH}, \mathrm{NO}_{2}, \mathrm{OCH}_{3}$,

Scheme 2. Synthesis of highly functionalized pyridine derivatives using 2- mercaptoethanol

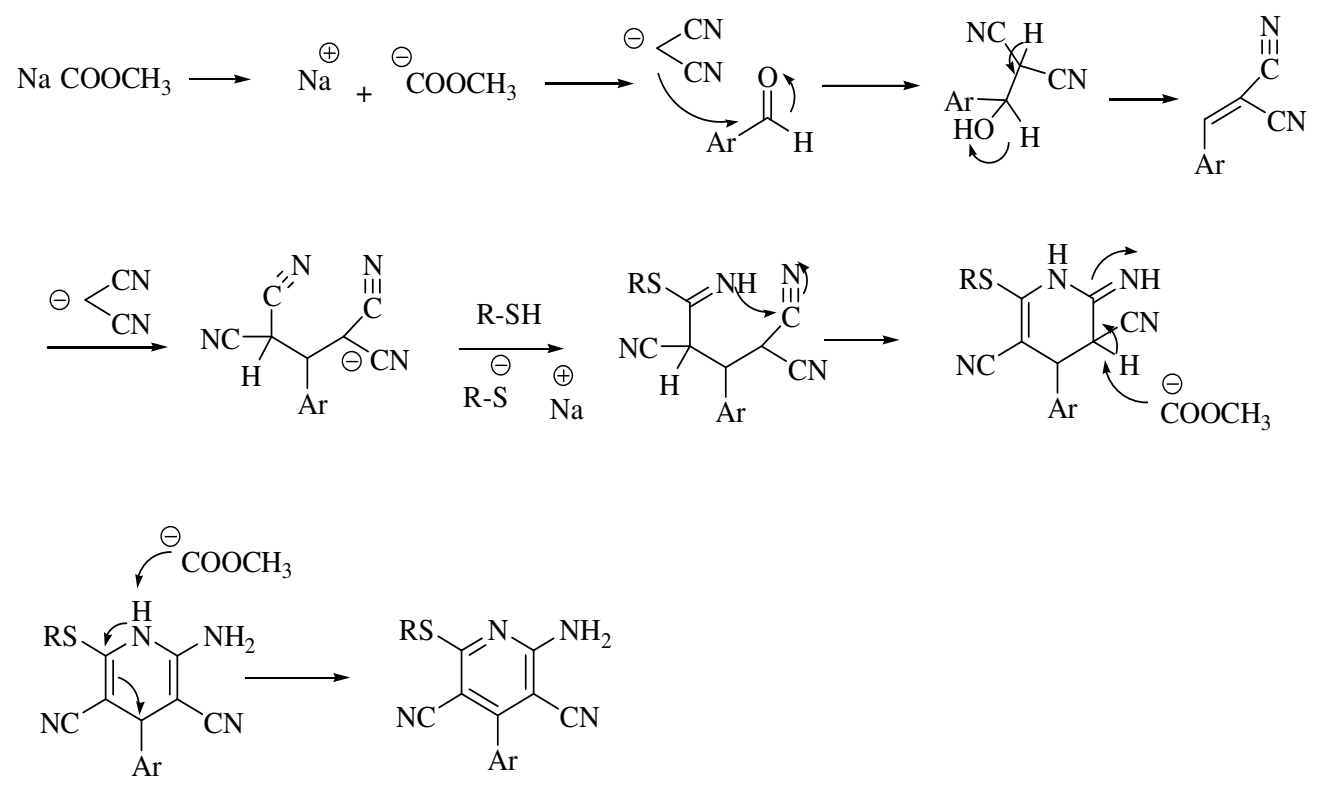

Scheme 3. Proposed mechanism for the synthesis of pyridine carbonitrile 
Table 1. Synthesis of 2-amino-3,5-dicarbonitrile-6-thio-pyridines using various catalysts $10 \mathrm{~mol} \%(280 \mathrm{~W})$

\begin{tabular}{|c|c|c|c|c|}
\hline Catalysts & Solvent & Conventional, $\mathrm{h}$ & Microwave, min & Isolated yield, \% \\
\hline $\mathrm{MeSO}_{3} \mathrm{H}$ & $\mathrm{CH}_{3} \mathrm{CN}$ & 8 & - & - \\
\hline $\mathrm{MeSO}_{3} \mathrm{H}$ & $\mathrm{EtOH}$ & - & 10 & 5 \\
\hline $\mathrm{BF}_{3}$ & $\mathrm{EtOH}$ & - & 8 & - \\
\hline $\mathrm{HBF}_{4} \cdot \mathrm{SiO}_{2}$ & $\mathrm{EtOH}$ & 8 & - & - \\
\hline $\mathrm{HBF}_{4} \cdot \mathrm{SiO}_{2}$ & - & - & 5 & 15 \\
\hline $\mathrm{HBF}_{4} \cdot \mathrm{SiO}_{2}$ & - & 3 & - & - \\
\hline $\mathrm{H}_{3} \mathrm{PW}_{12} \mathrm{O}_{40}$ & $\mathrm{EtOH}$ & 8 & - & - \\
\hline Alum & $\mathrm{H}_{2} \mathrm{O}$ & 8 & - & - \\
\hline $\mathrm{NH}_{2} \mathrm{PO}_{4}$ & $\mathrm{H}_{2} \mathrm{O}$ & 8 & - & - \\
\hline $\mathrm{NH}_{2} \mathrm{PO}_{4}$ & $\mathrm{EtOH}$ & 8 & - & 20 \\
\hline $\mathrm{KH}_{2} \mathrm{PO}_{4}$ & $\mathrm{H}_{2} \mathrm{O}$ & 8 & - & 13 \\
\hline $\mathrm{TBABr}$ & $\mathrm{H}_{2} \mathrm{O}$ & 8 & - & 15 \\
\hline $\mathrm{TBABr}+\mathrm{K}_{2} \mathrm{CO}_{3}$ & $\mathrm{H}_{2} \mathrm{O}$ & 8 & - & 20 \\
\hline $\begin{array}{l}\text { Sodium lauryl } \\
\text { sulphate }\end{array}$ & $\mathrm{H}_{2} \mathrm{O}$ & 8 & - & 10 \\
\hline L-Proline & $\mathrm{EtOH}$ & 8 & - & 25 \\
\hline $\mathrm{ZnO}$ & $\mathrm{EtOH}$ & 8 & - & 15 \\
\hline $\mathrm{CH}_{3} \mathrm{COONa}$ & $\mathrm{EtOH}$ & 2 & - & 55 \\
\hline $\mathrm{CH}_{3} \mathrm{COONa}$ & $\mathrm{EtOH}$ & - & 3 & 69 \\
\hline $\mathrm{CH}_{3} \mathrm{COONa}$ & $\mathrm{MeOH}$ & - & 3 & 78 \\
\hline Amberlyst & $\mathrm{EtOH}$ & 8 & - & - \\
\hline
\end{tabular}

Table 2. Synthesis of 2-amino-3, 5-dicarbonitrile-6-thio-pyridines in the presence of different solvents using sodium acetate $(10 \mathrm{~mol} \%)$ as a catalyst at $280 \mathrm{~W}$

\begin{tabular}{cccc}
\hline Entry & Solvent & Time, min & Yield, $\%^{\mathrm{a}}$ \\
\hline 1 & - & 1.3 & 65 \\
2 & Ethanol & 3 & 68 \\
3 & Ethanol 50\% & 4 & 55 \\
4 & Ethanol 10\% & 3 & 25 \\
5 & Water & 4 & 20 \\
6 & Methanol & 3 & 78 \\
7 & Acetonitrile & 4 & 58 \\
\hline
\end{tabular}

${ }^{a}$ Yields refer to the pure isolated products

Table 3. Effect of concentrations of catalyst

\begin{tabular}{cccc}
\hline Entry & Sodium acetate, mol\% & Time, min & Yield, $\%^{\mathrm{a}}$ \\
\hline 1 & 5 & 4 & 52 \\
2 & 7.5 & 3.5 & 60 \\
3 & 10 & 3 & 78 \\
4 & 12 & 3 & 89 \\
5 & 15 & 2.5 & 80 \\
\hline
\end{tabular}

${ }^{a}$ Yields refer to the pure isolated products 
Table 4. Synthesis of 2-amino-3, 5-dicarbonitrile-6-thio-pyridines

\begin{tabular}{cccccc}
\hline Entry & $\mathrm{Ar}$ & $\mathrm{CH}_{2}$ & Time, min & Yield, $\%^{\mathrm{a}}$ & M.P. ${ }^{0} \mathrm{C}[\mathrm{Ref}]^{\mathrm{b}}$ \\
\hline 1 & $\mathrm{C}_{6} \mathrm{H}_{5}$ & - & 3.0 & 89 & $216-217^{[14]}$ \\
2 & $4-\mathrm{Cl}^{\mathrm{C}} \mathrm{C}_{6} \mathrm{H}_{4}$ & - & 3.0 & 92 & $223-224^{[15]}$ \\
3 & $4-\mathrm{OCH}_{3}-\mathrm{C}_{6} \mathrm{H}_{4}$ & - & 4.0 & 91 & $241-243^{[15]}$ \\
4 & $4-\mathrm{NO}_{2}-\mathrm{C}_{6} \mathrm{H}_{4}$ & - & 5.0 & 85 & $286-287^{[14]}$ \\
5 & $4-\mathrm{OH}-\mathrm{C}_{6} \mathrm{H}_{4}$ & - & 4.5 & 86 & $312-314^{[14]}$ \\
7 & $2-\mathrm{OCH}{ }_{3}-\mathrm{C}_{6} \mathrm{H}_{4}$ & - & 6.0 & 82 & $280-282$ \\
8 & $4-\mathrm{OH}-3-\mathrm{OCH}_{3}-\mathrm{C}_{6} \mathrm{H}_{3}$ & - & 5.5 & 87 & $216-218^{[15]}$ \\
9 & $3-\mathrm{OH}-4-\mathrm{OCH}_{3}-\mathrm{C}_{6} \mathrm{H}_{3}$ & - & 5.5 & 84 & $274-276^{[14]}$ \\
10 & $\mathrm{C}_{6} \mathrm{H}_{5}-\mathrm{CH}_{2}-\mathrm{CH}_{2}$ & 1 & 5.0 & 82 & $270-271$ \\
11 & $3,4-\left(\mathrm{OCH}_{3}\right)_{2}-\mathrm{C}_{6} \mathrm{H}_{4}$ & - & 4.0 & 87 & $227-229^{[15]}$ \\
12 & $3,4,5-\left(\mathrm{OCH}_{3}\right)_{3}-\mathrm{C}_{6} \mathrm{H}_{4}$ & - & 3.0 & 89 & $236-238^{[14]}$ \\
13 & $2-\mathrm{Furyl}_{14}$ & - & 5.0 & 82 & $176-178^{[15]}$ \\
15 & $2-\mathrm{NO}_{2}-\mathrm{C}_{6} \mathrm{H}_{4}$ & - & 12.0 & 62 & $290-292$ \\
16 & $4-\mathrm{NO}_{2}-\mathrm{C}_{6} \mathrm{H}_{4}$ & - & 7.0 & 81 & $231-233$ \\
17 & $4-\mathrm{OH}-3-\mathrm{OCH}_{3}-\mathrm{C}_{6} \mathrm{H}_{3}$ & - & 8.5 & 78 & $208-210$ \\
18 & $4-\mathrm{OCH}_{3}-\mathrm{C}_{6} \mathrm{H}_{4}$ & - & 8.0 & 85 & $158-159$ \\
19 & $\mathrm{C}_{6} \mathrm{H}_{5}$ & - & 8.0 & 81 & $148-150$ \\
& $4-\mathrm{Cl}_{6}-\mathrm{C}_{6} \mathrm{H}_{4}$ & - & 7.0 & 87 & $167-169^{[10]}$ \\
\hline
\end{tabular}

${ }^{a}$ Yields refer to the pure isolated products

${ }^{b}$ All known products have been reported previously in the literature and were characterized by comparison of IR and NMR spectra with authentic samples. All new compounds characterized by melting point, IR, NMR $\left({ }^{1} \mathrm{H}\right.$ and $\left.{ }^{13} \mathrm{C}\right)$ and elemental analyses

\section{Spectral data and elemental analysis for new compounds}

2-Amino-4-(2-methoxyphenyl)-6-(phenylthio) pyridine-3, 5-dicarbonitrile (entry 7)

${ }^{1} \mathrm{H}$ NMR (200MHz, DMSO-d6) $\delta: 7.83$ (brs, $\left.\mathrm{NH}_{2}, 2 \mathrm{H}\right), 7.61-7.16(\mathrm{~m}, \mathrm{Ar}-9 \mathrm{H}), 3.86\left(\mathrm{~s}, \mathrm{OCH}_{3}\right)$; ${ }^{13} \mathrm{C}$ NMR (50MHz,DMSO-d6) $\delta: 55.41,72.0,83.23,116.82,120.68,129.65,159.0,159.81,190.36$; IR(KBr):3406,3328,3230,2961,2214,2363,2214,2164,1551,1467,1489,1318,1247,1150,1029, 834,788,259,706,579,413; $\mathrm{C}_{20} \mathrm{H}_{14} \mathrm{~N}_{4} \mathrm{OS}$; Anal.Calcd.for C, 67.02; H, 3.94; N, 15.63; S, 8.95; Found C, 67.07; H, 3.98; N, 15.67; S, 8.99.

\section{2-Amino-4-phenethyl-6-(phenylthio) pyridine-3, 5-dicarbonitrile (entry10)}

${ }^{1} \mathrm{H}$ NMR(200MHz, DMSO-d6)8:6.91(brs, $\left.\mathrm{NH}_{2}, 2 \mathrm{H}\right), 7.69-7.41(\mathrm{~m}, \mathrm{Ar}-10 \mathrm{H}), 3.24\left(\mathrm{~s}, \mathrm{CH}_{2}, 4 \mathrm{H}\right)$; ${ }^{13} \mathrm{C}$ NMR(50MHz,DMSO-d6) $\delta: 26.41, \quad 30.49,70.70,81.13,114.38,114.96,116.22,126.16$, 128.67,129.23,157.97,158.66,161.32; IR(KBr):3422, 3000, 2727, 2362, 1720, 1676, 1456, $1377,1303,1205,1154,1077,964,722 ; \mathrm{C}_{21} \mathrm{H}_{16} \mathrm{~N}_{4} \mathrm{~S}$, Anal.Calcd.for $\mathrm{C}, 70.76 ; \mathrm{H}, 4.52 ; \mathrm{N}, 15.72 ; \mathrm{S}$, 9.00; Found C, 70.86; H, 4.58; N, 15.78;S, 9.05.

\section{2-Amino-4-(2-nitrophenyl)-6-(phenylthio) pyridine-3, 5-dicarbonitrile (entry14)}

${ }^{1} \mathrm{H}$ NMR (200MHz, DMSO-d6) $8: 8.42$ (brs, $\left.\mathrm{NH}_{2}, 2 \mathrm{H}\right), 802-7.65(\mathrm{~m}, \mathrm{Ar}-9 \mathrm{H}) ;{ }^{13} \mathrm{C} \mathrm{NMR}(50 \mathrm{MHz}$,

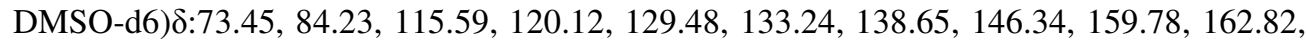
160.32;IR(KBr):3404, 3323，3153，3132，3078，2754，2218,1685,1660,1610,1579,1477, $1435,1325,1257,1151,1085 ; \mathrm{C}_{19} \mathrm{H}_{11} \mathrm{~N}_{5} \mathrm{O}_{2} \mathrm{~S}$ Anal.Calcd.for C, 61.12; H, 2.97; N, 18.76; S, 8.59; Found C, 61.14; H, 2.94; N, 8.81; S, 8.63. 
2-(2-Hydroxyethylthio)-6-amino-4-(4-nitrophenyl)pyridine-3,5-dicarbonitrile(entry 15)

${ }^{1} \mathrm{H}$ NMR(200MHz, DMSO-d6) $8: 3.32\left(\mathrm{~m}, 2 \mathrm{H}, \mathrm{CH}_{2}\right), 3.7\left(\mathrm{~m}, 2 \mathrm{H}, \mathrm{CH}_{2}\right), 5.0(\mathrm{brs}, 1 \mathrm{H}, \mathrm{OH}), 7.8$ (d, Ar-H,2H), 8.2(brs, 2H, NH (b. $^{2}$ 8.6(d, Ar-H, 2H); IR(KBr): 3477, 3427, 3327, 2879, 2218, 1680, 1552, 1518, 1411,1352,1267,1026, 956, 852; $\mathrm{C}_{15} \mathrm{H}_{1} 1 \mathrm{~N}_{5} \mathrm{O}_{3} \mathrm{~S}$ Anal.Calcd.for C, 52.78; $\mathrm{H}$, $3.25 ; \mathrm{N}, 20.52 ; \mathrm{S}, 9.39$; Found C, 52.75; H, 3.31; N, 20.57; S, 9.47.

2-(2-Hydroxyethylthio)-6-amino-4-(4-hydroxy-3-methoxyphenyl)pyridine-3,5-dicarbonitrile(entry16)

${ }^{1} \mathrm{H}$ NMR (200MHz, DMSO-d6) $8: 3.32\left(\mathrm{~m}, 2 \mathrm{H}, \mathrm{CH}_{2}\right), 3.7\left(\mathrm{~m}, 2 \mathrm{H}, \mathrm{CH}_{2}\right), 3.8\left(\mathrm{~s}, 3 \mathrm{H}, \mathrm{OCH}_{3}\right), 5.1$ (brs, 1H,OH), 7.1(brs, 1H,OH), 7.5-7.8(m, Ar-H, 3H), 7.9(brs, 2H, $\left.\mathrm{NH}_{2}\right)$; IR( $\left.\mathrm{KBr}\right): 3475,3371$, 2843, 2210,1926, 1680, 1566, 1514, 1406, 1307, 1168, 1021, 956, 850, 663; $\mathrm{C}_{16} \mathrm{H}_{14} \mathrm{~N}_{4} \mathrm{O}_{3} \mathrm{~S}$ Anal. Calcd. C, 56.13; H, 4.12; N, 16.36; O, 14.02; S, 9.37; Found C, 56.10; H, 4.22; $\mathrm{N}, 16.45 ; \mathrm{S}, 9.37$.

2-(2-Hydroxyethylthio)-6-amino-4-(4-methoxyphenyl) pyridine-3, 5-dicarbonitrile, (entry17)

${ }^{1} \mathrm{H}$ NMR (200MHz,DMSO-d6) $\delta: 3.31\left(\mathrm{~m}, 2 \mathrm{H}, \mathrm{CH}_{2}\right), 3.63\left(\mathrm{~m}, 2 \mathrm{H}, \mathrm{CH}_{2}\right), 3.8\left(\mathrm{~s}, 3 \mathrm{H}, \mathrm{OCH}_{3}\right), 5.0$ (brs, 1H,OH), 7.0(d,2H,Ar-H), 7.4(d,2H,Ar-H), 7.9(brs,2H,NH ( $_{2}$; IR( KBr):3421,3340, 2840, $2214,1901,1880,1647,1550,1514,1419,1253,1186,1039,956,779 ; \mathrm{C}_{16} \mathrm{H}_{14} \mathrm{~N}_{4} \mathrm{O}_{2}$ : Anal. Calcd. C, 58.88; H, 4.32; N, 17.17;S, 9.82 ; Found C, 58.94; H, 4.34; N, 17.15; S, 9.81

2-(2-Hydroxyethylthio)-6-amino-4-phenylpyridine-3,5-dicarbonitrile (entry18)

${ }^{1} \mathrm{H}$ NMR (200MHz,DMSO-d6) $8: 3.33\left(\mathrm{~m}, 2 \mathrm{H}, \mathrm{CH}_{2}\right), 3.66\left(\mathrm{~m}, 2 \mathrm{H}, \mathrm{CH}_{2}\right), 5.1(\mathrm{brs}, 1 \mathrm{H}, \mathrm{OH}), 7.5-$ 7.8(m,Ar-H,5H), 8.1(brs,2H, $\mathrm{NH}_{2}$ ); IR(KBr):3440,3356,2847, 2212,1915,1896,1642,1548, $1523,1414,1190,1023,955 ; \mathrm{C}_{15} \mathrm{H}_{12} \mathrm{~N}_{4} \mathrm{OS}$ :Anal. Calcd. C, 60.79; H, 4.08; N, 18.91;S,10.82; Found C, 60.80; H, 4.06; N, 18.94; S, 10.84 .

\section{Conclusion}

An efficient and environmentally benign strategy for the synthesis of highly functionalized pyridine derivatives is developed. The method offers several advantages including high yield of products, short reaction time, easily availability of catalyst, ease of work-up and low-cost.

\section{Acknowledgment}

Authors are thankful to University Grants Commission, New Delhi, for financial Assistance and Nyala University-Sudan for their support.

\section{References}

1. Zhu J and Bienayme H, Multicomponent Reactions; Wiley-VCH: Weinheim, 2005.

2. Ugi I, Dömling A and Hörl W, Multicomponent Reactions in Organic Chemistry Endeavour, 1994, 18(3), 115-122; DOI:10.1016/S0160-9327(05)80086-9

3. Chen H, Zhang W, Tam R and Raney A K, PCT Int Appl., 2005, WO, 058315 A120050630

4. Perrier V, Wallace A C, Kaneko K, Safar J, Prusiner S B and Cohen F E, Natl Acad Sci U.S.A., 2000, 97, 6073-6078.

5. Levy S B, Alekshun M N, Podlogar B L, Ohemeng K, Verma A K, Warchol T, Bhatia B, Bowser T and Grier M, U.S. Patent Appl., 2005, 24678 A1 20050609. 
6. Anderson D R, Stehle N W, Kolodziej S A and Reinhard E, J PCT Int Appl., 2004, WO 055015 A1 20040701.

7. Harada H, Watanuki S, Takuwa T, Kawaguch K, Okazaki T, Hirano Y and Saitoh C, PCT Int Appl., 2002, WO, 006237 A1 20020124.

8. Beukers M W, Chang L C W, Frijtag Drabbe Künzel J K, Mulder-Krieger T, Spanjersberg R F, Brussee J and Ijzerman A P, J Med Chem., 2004, 47(15), 37073709; DOI:10.1021/jm049947s

9. Fredholm B B, Ijzerman A P, Jacobson K A and Klotz K N Linden, J Pharmacol Rev., 2001, 53, 527-552.

10. Evdokimov N M, Kireev S A, Yakovenko A A, Antipin M Y, Magedov I V and Kornienko A, J Org Chem., 2007, 72(9), 3443-3453; DOI:10.1021/jo070114u

11. Ranu B C, Jana R and Sowmiah S, J Org Chem., 2007, 72(8), 3152-3154; DOI:10.1021/jo070015g

12. Mamgain R, Singh R and Rawat D S, J Heterocycl Chem., 2009, 46(1), 69-73; DOI:10.1002/jhet.32

13. Sridhar M, Ramanaiah B C, Narsaiah C, Mahesh B, Kumaraswamy M, Mallu K K R, Ankathi V M and Rao P S, Tetrahedron Lett., 2009, 50(30), 3897-3901; DOI:10.1016/j.tetlet.2011.05.079

14. Evdokimov N M, Magedov I V, Kireev A S and Kornienko A, Org Lett., 2006, 8(5), 899-902; DOI:10.1021/o1052994+

15. Shinde P V, Sonar S S, Shingate B B and Shingare M S, Tetrahedron Lett., 2010, 51(9), 1309-1312; DOI:10.1016/j.tetlet.2009.12.146 